TITLE:

\title{
Evaluation method of lead measurement accuracy of gears using a wedge artefact
}

\section{AUTHOR(S):}

Komori, Masaharu; Takeoka, Fumi; Kubo, Aizoh; Okamoto, Kazuhiko; Osawa, Sonko; Sato, Osamu; Takatsuji, Toshiyuki

\section{CITATION:}

Komori, Masaharu ... [et al]. Evaluation method of lead measurement accuracy of gears using a wedge artefact. Measurement Science and Technology 2009, 20(2): 025109.

\section{ISSUE DATE:}

2009-01-20

URL:

http://hdl.handle.net/2433/210473

\section{RIGHT:}

This is an author-created, un-copyedited version of an article accepted for publication in 'Measurement Science and Technology'. The publisher is not responsible for any errors or omissions in this version of the manuscript or any version derived from it. The Version of Record is available online at http://dx.doi.org/10.1088/0957-0233/20/2/025109.; This is not the published version. Please cite only the published version.; この論文は出版社版でありません。引用の際には出 版社版をご確認ざ利用ください。 


\title{
Evaluation method of lead measurement accuracy of gears using a wedge artefact
}

\author{
Masaharu KOMORI ${ }^{1,3}$, Fumi TAKEOKA ${ }^{1}$, Aizoh KUBO ${ }^{1}$, Kazuhiko OKAMOTO ${ }^{1}$, Sonko OSAWA ${ }^{2}$, Osamu SATO $^{2}$ and \\ Toshiyuki TAKATSUJI ${ }^{2}$ \\ 1 Department of Mechanical Engineering and Science, Kyoto University, Yoshidahonmachi, Sakyo-ku, Kyoto-shi, Kyoto 606-8501, \\ Japan
}

Tel: 81-75-753-5858

Fax: 81-75-753-5858

E-mail: komorim@me.kyoto-u.ac.jp

2 Dimensional Standards Section, National Metrology Institute of Japan, AIST, Tsukuba, Ibaraki, 305-8563, Japan

Tel: 81-29-861-4041

Fax: 81-29-861-4042

E-mail: toshiyuki.takatsuji@aist.go.jp

3 Corresponding author

\begin{abstract}
The reduction of the vibration and noise of gears is an important issue in mechanical devices such as vehicles and wind turbines. The characteristics of the vibration and noise of gears are markedly affected by deviations of the tooth flank form of micrometer order; therefore, strict quality control of the tooth flank form is required. The accuracy of the lead measurement for a gear-measuring instrument is usually evaluated using a master gear or a lead master. However, it is difficult to manufacture masters with high accuracy because the helix is a complicated geometrical form. In this report, we propose a method of evaluating a gear-measuring instrument using a wedge artefact, which includes a highly precise plane surface. The concept of the wedge artefact is described and a mathematical model of the measuring condition of the wedge artefact is constructed. Theoretical measurement results for the wedge artefact are calculated. The wedge artefact is designed and produced on the basis of the theoretical measurement results. A measurement experiment using the wedge artefact is carried out and its effectiveness is verified.
\end{abstract}

Keywords: artefact, gear, lead measurement, evaluation, gear-measuring instrument 


\section{Introduction}

The reduction of gear vibration and noise is one of the important issues in automobiles because it is a factor influencing the attractiveness of automobiles [1-2]. Because the vibration and noise of gears are affected by deviations of the tooth flank form of micrometer order, severe quality control is required for the tooth flank form of gears. Various types of measuring methods exist for evaluating the tooth flank form of gears [3-9]. In many factories, a specialized measuring machine for gears [9] and a coordinate measuring machine [10] are used, although other methods such as fringe projection [3], holographic interferometry [11], optical measurement [12], and the use of a laser tracker [13-14], have been proposed. For the tooth flank form, a gear-measuring instrument (a specialized machine for measuring gears) is often used for quality control because of its ease of use and high measurement speed. Therefore, a highly accurate gear-measuring instrument is very important for realizing the advanced quality control of gears. For high-class cars which require low gear noise, even undulation of $2 \mu \mathrm{m}$ on tooth flank has an effect on the quality of gear noise. Thus, the accuracy of $1 \mu \mathrm{m}$ or higher is required for gear-measuring instrument. However, it is thought that the accuracy of current gear-measuring instruments is at the several-micrometer level and does not satisfy the required accuracy.

To verify the accuracy of gear-measuring instruments, a method using laser interferometry [15] has been reported but, in most cases, an artefact (master gauge) with high accuracy is used. In principle, the accuracy of the gear-measuring instrument does not exceed the accuracy of the artefact used for calibration; thus, high accuracy is required for the artefact. In many factories, a master gear [16], which has a similar shape to a common gear, is used to evaluate the gear-measuring instrument. In addition, to measure the profile of the teeth, various types of specialized master gauges exist that have an involute profile form (profile artefact or involute artefact), and, for lead measurement (the measurement in the tooth width direction), master gauges with a helicoid surface (lead artefact or helicoid artefact) exist [16-20]. However, the accuracy of these artefacts (master gauges) is not satisfactory because they are difficult to manufacture due to their geometrically complicated reference surface. To solve this problem for the measurement of the tooth profile, a ball artefact [21] has been proposed, which results in evaluation with small uncertainty because the ball can be manufactured with a very high form-accuracy of several tens of nanometers. A gear requires very high precision of the tooth lead form as well as the tooth profile form. However, a highly accurate artefact for evaluating the lead-measuring function of the measurement instrument has not been developed. In this study, an artefact with a plane is proposed to evaluate the lead measurement accuracy. A plane is taken as the reference surface because it can be manufactured with a high accuracy of several tens of nanometers. The concept of the artefact is proposed, a theoretical analysis and design are performed, and an evaluation method is developed. The artefact is then produced and its accuracy is calibrated. A measurement experiment using the proposed artefact is carried out and the effectiveness of the evaluation method is verified.

\section{Problems in evaluation of tooth lead measurement}




\subsection{Principle of tooth lead measurement}

In this study, a measuring instrument for a gear with an involute helicoid tooth flank is taken. Figure 1 shows the base cylinder and the involute helicoid surface of a gear. $T_{1} T_{2}$ is curve intersecting the involute helicoid and the cylindrical surface, and this curve represents a tooth lead. In the tooth lead measurement, the measuring probe is generally moved along $\mathrm{T}_{1} \mathrm{~T}_{2}$, and the difference between the theoretical helix form and the actual form on the gear tooth flank is outputted [9]. As shown in figure 1, the base circle radius of the measured helical gear is $r_{b}$ and the tooth width is $h$. The radius of the circle where the lead measurement is performed is $r_{m}$ and the helix angle on this cylinder is $\beta_{m}$. Figure 2 shows the development surface of the lead measurement cylinder. The angle between line $\mathrm{T}_{1} \mathrm{~T}_{2}$ and the gear axis (z axis) is the helix angle $\beta_{m}$. Various methods exist for realizing the motion of the measuring probe from $T_{1}$ to $T_{2}$. A common method is to rotate the gear synchronizing with the movement of the probe in the gear axial direction (z direction). This motion makes the relative motion of the probe follow a helical curve. This is a generating-type of tooth lead measurement method and, in this study, this measurement method is investigated. In this method, the relation between the amount $d z$ of probe movement in the axial direction and the rotational angle $d \theta$ of the measured gear is expressed in the following equation:

$$
r_{m} d \theta=d z \tan \beta_{m} .
$$

The difference between the theoretical helix form and the actual form is expressed in the direction of the line tangential to the base circle of the gear (dotted line in figure 1).

Figure 3 shows a schematic model of the gear-measuring instrument investigated in this study. A gear is set between the upper and lower fixing centres of the measuring instrument, and the probe sphere is placed in contact with the tooth flank. The tooth lead measurement is performed by moving the probe in the $\mathrm{z}$ direction and rotating the gear in accordance with equation (1). The probe sphere is displaced in the y axis direction depending on the actual form deviation of the measured tooth flank, and this displacement is detected using the displacement sensor. The y displacement of the probe sphere is plotted as a function of the $\mathrm{z}$ position of the probe on the output graph representing the measurement result.

\subsection{Current state and problems of master gauges}

The tooth lead measurement function of the gear-measuring instrument is evaluated using a master gauge with higher accuracy than the measuring instrument. The lead master shown in figure 4 is an example of a master gauge with a helix form on the reference surface [16-20]. The involute form or the straight form is used for the profile form of the lead master. On the other hand, in many factories, the accuracy evaluation of the gear-measuring instrument is performed using master gears [16], which are gears with very high accuracy. The tooth form of the master gear is almost identical to that of actual gears. However, both the lead master and the master gear are difficult to produce with high accuracy because the reference surface has a complicated three-dimensional curved surface. In addition, it is difficult to reduce the roughness and the 
undulation of the reference surface. Therefore, it is suggested that their accuracies are limited to about $1 \mu \mathrm{m}$ at best. The accuracies of the evaluation and calibration of a gear-measuring instrument do not exceed the accuracy of these master gauges. Because the deviations of the tooth flank form with micrometer order affect the vibration and noise performance of gears, greater accuracy is needed for the gear-measuring instrument. In this respect, the accuracy of current master gauges is insufficient.

\section{Wedge artefact}

To solve this problem, we propose an artefact for lead measurement whose reference surface is composed of a plane. This artefact is named the "wedge artefact".

\subsection{Fundamental concept of wedge artefact}

The basic concept of the proposed artefact is to use a simple geometric shape. Compared with a complex shape such as a helix or involute, a simple shape can be produced with high accuracy. In addition, highly accurate measuring instruments exist that can measure the dimensions and shape of objects with a simple shape. This leads to a small uncertainty value. In this study, a plane is used because it is a simple geometrical form that is partly similar to a helix form. Figure 5 shows a schematic diagram of the proposed wedge artefact. Plane $S_{1}$ is measured instead of the tooth flank by the lead measurement method. This plane can be made partially similar to the shape of the helix by selecting an appropriate angle of inclination of the plane.

Figure 6 shows a flowchart used to evaluate the accuracy of the gear-measuring instrument using the wedge artefact. First, the virtual measurement result (theoretical measurement curve) of the wedge artefact is calculated, where an ideal gear-measuring instrument without any errors is assumed. This theoretical lead measurement curve is compared with the actual lead measurement result, which is output from the actual gear-measuring instrument with some error, and the difference between these curves is considered as the accuracy of the gear-measuring instrument.

For a tooth profile measurement, a double-ball artefact (DBA) is proposed [21], for which a simple shape is also used. The DBA is composed of spheres and it is measured instead of an involute tooth flank. Also in this case, the theoretical curve and the actual measurement curve are compared, and the difference between them indicates the accuracy of the measuring instrument. The wedge artefact is similar to the DBA in the points that it has a simple shape and compares the theoretical curve and the actually measured curve to evaluate the gear-measuring instrument.

\subsection{Mathematical model of tooth lead measurement using wedge artefact}

Figure 7 shows the helix and the ellipse on the lead measurement cylinder. The ellipse in the figure corresponds to the curve intersecting the lead measurement plane $\left(S_{1}\right.$ in figure 5$)$ of the wedge artefact and the lead measurement cylinder. The gear-measuring instrument outputs the deviation of the actual tooth flank from the theoretical helix as the lead measurement result. Therefore, when the wedge artefact is measured, 
the deviation between the theoretical helix and the ellipse shown in figure 7 is output. Assuming a gear-measuring instrument without any error, a mathematical model for the lead measurement of the plane of the wedge artefact is constructed and the virtual output result of the measurement is analyzed in this section.

As shown in figure 8, a vector in the maximum-inclination direction of the lead measurement plane is composed of the $\mathrm{x}$ and $\mathrm{y}$ components of a normal vector to the lead measurement plane. The maximum-inclination cross-section plane is parallel to the $\mathrm{z}$ axis and the normal vector to the lead measurement plane. The wedge artefact is set up on the gear-measuring instrument as shown in figure 8, so that the maximum-inclination direction of the lead measurement plane is parallel to the $y$ axis of the measuring instrument. This state is called the basic posture.

Figure 9 shows the case where the nominal measurement point of the lead is located at the intersection of the lead measurement circle and the line $y=0$. This corresponds to the lead measurement on the base circle of the involute helicoid gear. On the basis of the methodology explained below, it is also possible to construct a mathematical model for the lead measurement at positions different from that shown in figure 9. $r_{m}$ is radius of the lead measurement circle, $\beta_{m}$ is the helix angle on the lead measurement cylinder, and $r_{p}$ is the radius of the probe sphere. The inclination angle of the lead measurement plane $S_{1}$ of the wedge artefact is assumed to be $\Phi$ (angle from the xy plane).

Figure 10 shows the state of the apparatus when the probe sphere is in contact with the lead measurement plane $S_{1}$ of the wedge artefact set in the basic posture on the gear-measuring instrument. A generating-type of gear-measuring instrument has a displacement sensor in the y direction and the probe sphere is displaced in the y direction. In this analysis, to consider the general state, we impose the condition that there is an offset in the sensing direction (y direction) between the probe sphere and the nominal measurement point. As shown in this figure, $\mathrm{P}_{0}$ is the position of the centre of the probe sphere in the basic posture, and $\mathrm{A}_{0}$ is the point intersecting the sensing direction line, which is parallel to the y axis and passes through $\mathrm{P}_{0}$, and the lead measurement plane $\mathrm{S}_{1}$ of the wedge artefact. $l$ is defined as the y coordinate of the horizontal line on the lead measurement plane passing through $\mathrm{A}_{0}$. The offset of the centre of the probe sphere is $l+\mathrm{A}_{0} \mathrm{P}_{0}$. Under this condition, the origin of the $\mathrm{z}$ axis is set at the height of the centre of the probe sphere.

Figure 11 shows the state of the apparatus after the rotary stage and the wedge artefact have rotated by $\theta$ from the state of the basic posture. For the tooth lead measurement, the probe sphere moves in the $\mathrm{z}$ direction synchronizing with the rotation of the rotary stage. As shown in the figure, $\mathrm{P}_{1}$ is the centre of the probe sphere after a rotation of $\theta, \mathrm{A}_{1}$ is the point intersecting the sensing direction line passing through $\mathrm{P}_{1}$ and the lead measurement plane $S_{1} \cdot Z_{a}$ is the displacement of the probe sphere in the $\mathrm{z}$ direction synchronizing with the rotation by $\theta$.

The state in the basic posture and that after the wedge artefact has rotated by $\theta$ are displayed in figure 12 . $\mathrm{A}_{0 \mathrm{p}}$ and $\mathrm{P}_{0 \mathrm{p}}$ are the projections of $\mathrm{A}_{0}$ and $\mathrm{P}_{0}$ onto the plane $\mathrm{z}=z_{a}$, respectively.

The tooth lead deviation $e$ after a rotation of $\theta$ is expressed as

$$
e=\mathrm{A}_{0 \mathrm{p}} \mathrm{A}_{1}+\mathrm{A}_{1} \mathrm{P}_{1}-\mathrm{A}_{0 \mathrm{p}} \mathrm{P}_{0 \mathrm{p}} \text {. }
$$

As shown in figure 13, the second and third terms on the right-hand side of this equation can be given as follows. 


$$
\begin{aligned}
& \mathrm{A}_{0 \mathrm{p}} \mathrm{P}_{0 \mathrm{p}}=\frac{r_{p}}{\sin \Phi} \\
& \mathrm{A}_{1} \mathrm{P}_{1}=\frac{r_{p}}{\sin \Phi} \frac{1}{\cos \theta}
\end{aligned}
$$

Next, an expression for $A_{0 p} A_{1}$ in equation (2) is needed. From figure 14, $A_{0 p} A_{1}$ is given as follows. $B$ is the point on the lead measurement plane after a rotation of $\theta$ and it has identical $\mathrm{x}$ and y coordinates to $\mathrm{A}_{0 \mathrm{p}}$.

$$
\begin{aligned}
\mathrm{A}_{0 \mathrm{p}} \mathrm{A}_{1} & =\mathrm{A}_{0 \mathrm{p}} \mathrm{C}_{1} \frac{1}{\cos \theta} \\
& =\frac{z_{b}-z_{a}}{\tan \Phi} \frac{1}{\cos \theta}
\end{aligned}
$$

The change in the height of the probe sphere $z_{a}$ is obtained by the following equation, by considering that its movement follows the theoretical form of the helix.

$$
z_{a}=r_{m} \theta \tan \left(\pi / 2-\beta_{m}\right)
$$

$z_{b}$ is obtained from the following equations obtained by considering figure 15.

$$
\begin{aligned}
& \mathrm{GK}=\left(r_{m}+l \tan \theta+\frac{l}{\sin \theta}\right) \sin \theta \\
& =r_{m} \sin \theta+l\left(\frac{\sin ^{2} \theta}{\cos \theta}+1\right) \\
& \mathrm{GJ}=\frac{l}{\cos \theta} \\
& \mathrm{JK}=\mathrm{GK}-\mathrm{GJ} \\
& =r_{m} \sin \theta+l\left(\frac{\sin ^{2} \theta}{\cos \theta}+1\right)-\frac{l}{\cos \theta} \\
& =r_{m} \sin \theta+l(1-\cos \theta) \\
& z_{b}=\mathrm{JK} \tan \Phi \\
& =\left(r_{m} \sin \theta+l(1-\cos \theta)\right) \tan \Phi
\end{aligned}
$$

The equation expressing the lead deviation in the measurement of the wedge artefact is obtained by substituting these equations into equation (5).

$$
e=\frac{1}{\tan \Phi} \frac{1}{\cos \theta}\left(\left(r_{m} \sin \theta-l(1-\cos \theta)\right) \tan \Phi-r_{m} \theta \tan \left(\pi / 2-\beta_{m}\right)\right)+\frac{r_{p}}{\sin \Phi} \frac{1}{\cos \theta}-\frac{r_{p}}{\sin \Phi}
$$

The value of $l$, which is related to the offset, when the centre of the probe sphere is located on the lead measurement circle and on the sensing direction line at the beginning of the measurement, is expressed by equation (12). Under this condition, the lead deviation $e$ is expressed as equation (13).

$$
\begin{gathered}
l=-\mathrm{A}_{0 \mathrm{p}} \mathrm{P}_{0 \mathrm{p}} \\
=-\frac{r_{p}}{\sin \Phi} \\
e=\frac{1}{\tan \Phi} \frac{1}{\cos \theta}\left(\left(r_{m} \sin \theta-\frac{r_{p}}{\sin \Phi}(1-\cos \theta)\right) \tan \Phi-r_{m} \theta \tan \left(\pi / 2-\beta_{m}\right)\right)+\frac{r_{p}}{\sin \Phi} \frac{1}{\cos \theta}-\frac{r_{p}}{\sin \Phi}
\end{gathered}
$$




\subsection{Virtual measurement result of wedge artefact}

A virtual measurement result (theoretical measurement curve in figure 6) is shown in figure 16 using equation (13). The solid line indicates the case when the inclination angle $\Phi$ (see figure 13) of the lead measurement plane of the wedge artefact is 70.2 degrees, the radius of the lead measurement circle is $50 \mathrm{~mm}$, the helix angle on the lead measurement cylinder is 20 degrees, and the radius of the probe sphere is $1 \mathrm{~mm}$. This result shows that the virtual measurement curve is an S-shaped curve when the inclination angle of the lead measurement plane is selected such that the difference between the lead measurement plane $S_{1}$ and the helicoid becomes small. The measurement stroke of the displacement sensor of the gear-measuring instrument is usually limited, for example, within the range of $\pm 100 \mu \mathrm{m}$. Figure 16 shows that under such a limited measurement stroke of the sensor as above, it is possible to measure over a range of $85 \mathrm{~mm}$ in the tooth width direction. This result indicates that the measurement range of the wedge artefact in the tooth width direction is sufficiently wide compared with the tooth width of common mass-production gears such as those for automobiles. From these results, it is verified that the concept of using a plane as a reference surface instead of a helicoid surface is basically effective.

The broken line in figure 16 shows another virtual measurement result of the same wedge artefact, where the radius of the lead measurement circle is $200 \mathrm{~mm}$, the helix angle is 19.9 degrees, and the radius of the probe sphere is $1 \mathrm{~mm}$. This result shows that it is possible to measure a wide range of tooth width even on such a large measurement circle. In other words, the measurement of gears with a range of specifications can be treated using only one wedge artefact. The inclination angle of the lead measurement plane of the wedge artefact is mainly designed on the basis of the helix angle on the lead measurement cylinder. Therefore, the wedge artefact can be used to evaluate the measurement of gears of various specifications, provided the helix angle on the lead measurement cylinder remains almost constant. A conventional master gear is used to evaluate the measurement in only one gear specification, and the corresponding base circle radius and the helix angle on the base cylinder are limited to one value. To evaluate the measurement of a gear with another specification, another master gear is needed. The ability to treat gears of various specifications is an advantage of the wedge artefact.

\section{Design and production of wedge artefact}

\subsection{Design}

The wedge artefact is schematically shown in figure 5. Plane $S_{1}$ is the lead measurement plane, and the gear-measuring instrument is evaluated by measuring plane $S_{1}$ instead of a gear tooth flank. Planes $S_{2}, S_{3}$, and $S_{4}$ are used to confirm the posture of the wedge artefact when it is set up on the gear-measuring instrument. The angle of plane $S_{1}$ to the xy plane or the $\mathrm{z}$ axis of the measuring instrument is the most important factor. In this study, this angle is designed so as to make the measurement range in the tooth width direction as wide as possible while satisfying the limitation concerning the measurement stroke of the 
displacement sensor of the gear-measuring instrument. The theoretical measurement curves are investigated using equation (13) to make the measurement range as wide as possible, assuming that a lead measurement is performed for a measurement circle radius of $49 \mathrm{~mm}$, a helix angle on the measurement cylinder of 30 degrees, and a radius of the probe sphere of $3 \mathrm{~mm}$. As a result, the angle between planes $S_{1}$ and $S_{2}$ is designed to be 29.83 degrees, in other words, $\Phi=60.17$ degrees (see figure 13). Figure 17 shows the theoretical curve obtained when this artefact is measured. It is clarified that the measurable range in the tooth width direction is about $50 \mathrm{~mm}$ when the measurement stroke of the displacement sensor is $\pm 100 \mu \mathrm{m}$. The parameters of the wedge artefact, $w, d, h_{1}$, and $h_{2}$ in figure 5 , were designed to be $50 \mathrm{~mm}, 90 \mathrm{~mm}, 130 \mathrm{~mm}$, and $10 \mathrm{~mm}$, respectively.

\subsection{Production of wedge artefact}

Cemented carbide is used as the material of the wedge artefact because of its high hardness. The lead measurement plane $S_{1}$ is lapped after grinded because low roughness and few undulations are important as well as its flatness. The other planes are grinded without lapping because only their flatness is important. Figure 18 shows a photograph of the produced wedge artefact. Not only plane $S_{1}$ but also the other planes have a mirror-like condition, and the roughness is low.

\section{Calibration of wedge artefact}

\subsection{Measurement using coordinate measuring machine}

The accuracy of the produced artefact is evaluated. Each plane is measured using a coordinate measuring machine (Leitz PMM866) at the National Metrology Institute of Japan. Table 1 shows the measured flatness of each plane of the wedge artefact. The lead measurement plane $S_{1}$, the most important plane of the wedge artefact, has a flatness of less than $1 \mu \mathrm{m}$. This flatness value applies to the whole plane. Actually, in the tooth lead measurement, only a limited area of plane $S_{1}$ is used, and therefore it is thought that the flatness is actually much smaller than $1 \mu \mathrm{m}$ considering the actually measured area of plane $\mathrm{S}_{1}$. The angles between planes $S_{1}$ and $S_{2}$, planes $S_{1}$ and $S_{3}$, and planes $S_{1}$ and $S_{4}$ were measured to be 29.83418 degrees, 89.99485 degrees, and 90.00568 degrees, respectively. The angle between planes $S_{1}$ and $S_{2}$ was almost exactly the designed value.

\subsection{Optical interference measurement}

The lead measurement plane was successfully measured using an optical interferometer (ZYGO GPI), which indicated that the roughness and undulation of the plane were small. The measured flatness of the lead measurement plane $S_{1}$ is about $0.5 \mu \mathrm{m}$. As previously stated, it is thought that the flatness of the plane is higher if only the actually used area of the lead measurement plane is considered. These results indicate that the accuracy of the surface form of wedge artefact is higher than that of current master gears. Furthermore, by using planes for the artefact, it is possible to accurately calibrate the artefact using a measuring instrument 
with sufficiently high accuracy. This may reduce the uncertainty included in the artefact.

\section{Experiment of measurement of wedge artefact using gear-measuring instrument}

\subsection{Assumption of evaluation of gear-measuring instrument using wedge artefact}

The gear-measuring instrument has various error factors but the most important one for the generating-type of lead measurement is the synchronous accuracy between the rotation of the rotary stage and the movement of the probe sphere in the $\mathrm{z}$ direction. In this study, the main purpose of evaluating the lead measurement is to analyze this synchronous accuracy, and a method of evaluating the measuring instrument is developed on this basis. Therefore, the straightness of the xyz stage of the gear-measuring instrument, the perpendicularity of the $\mathrm{x}, \mathrm{y}$, and $\mathrm{z}$ axes, and the parallelism of the $\mathrm{z}$ axis and the axis of the rotary stage must have already been calibrated.

\subsection{Measurement procedure}

The procedure for measuring the wedge artefact is as follows (see figure 19(a)).

(1) Setting the wedge artefact in the basic posture (refer to figure 8).

a) The artefact is set up on the rotary stage in approximately the basic posture. As shown in figure 19, a cube is used to adjust the $\mathrm{z}$ position of the wedge artefact.

b) A magnetic base, a universal arm, and a dial gauge are installed on the xyz stage of the gear-measuring instrument.

c) It is confirmed that plane $S_{4}$ is parallel to the $y$ axis by placing the dial gauge in contact with plane $\mathrm{S}_{4}$ and moving it in the y direction using the y stage. If it is not parallel, the rotary stage of the measuring instrument is rotated, and this process is repeated.

d) The parallelism of plane $S_{4}$ and the $\mathrm{z}$ axis is confirmed by placing the dial gauge in contact with plane $\mathrm{S}_{4}$ and moving it in the $\mathrm{z}$ direction.

e) The parallelism of plane $S_{2}$ to the $\mathrm{z}$ axis is confirmed, by placing the dial gauge in contact with plane $\mathrm{S}_{2}$ and moving it in the $\mathrm{z}$ direction.

(2) Setting the probe position

The probe is moved so that the condition shown in figure 10 is realized after the probe has been placed in contact with plane $S_{1}$ and the output of the displacement sensor has become zero. In this case, point $A_{0}$ is located at $\mathrm{x}=r_{m}$ and $\mathrm{y}=0$, and the centre of the probe is at $\quad x=r_{m}$ and $y=\frac{r_{p}}{\sin \Phi}$.

(3) Lead measurement (see figure 19(b))

The lead measurement of the wedge artefact is performed by rotating the artefact while moving the probe sphere in the $\mathrm{z}$ direction similar to the usual method of lead measurement.

Note that it is desirable that the output of probe (displacement sensor) should be calibrated before measuring wedge artefact, because the wide measurement range of the displacement sensor is used in the measurement 
of wedge artefact.

\subsection{Measurement experiment}

The measurement of the wedge artefact is conducted in accordance with the above-mentioned procedure. Table 2 shows the parameters in the measurement experiment. In the experiment, different helix angles on the lead measurement cylinder are input into the gear-measuring instrument. In experiment No.8, the measurement circle radius is changed. The radius of the probe sphere is $3 \mathrm{~mm}$.

It was confirmed that it is possible to perform the whole experimental procedure without any serious problems. However, in some cases, the root of the probe interfered with the side edge of the lead measurement plane during lead measurement when the position of the probe was close to the rotation axis. Therefore, it is clarified that the area near the side edge of the lead measurement plane should be used.

\subsection{Analysis of the result of measurement experiment}

The actual measurement result and the theoretical curve are shown in figure 20 for experiment No.1. For the lead form, the shape of the lead deviation curve is more important than the absolute value of the measured deviation, because it affects the performance of the gear. Therefore, the position of the actually measured curve in the output graph has little meaning. Thus, in this analysis, the theoretical curve is moved to the position that most closely fits the measurement curve without changing its shape.

The measured curve is an S-shaped curve similar to the theoretical curve. The same result was achieved in the other experiments (No.2-8). Figure 21 shows the deviation of the curve corresponding to the difference between the measured curve and the theoretical curve in figure 20.

\subsection{Discussion on experimental result}

It is possible to evaluate the accuracy of the gear-measuring instrument by analyzing the deviation curve in figure 21. The high-frequency component is included in the deviation curve. The conventional master gear or the lead master has some roughness and undulation with a high-frequency component on the measured surface. Therefore, in the case of evaluating the gear-measuring instrument using the conventional master gear or lead master, if a high-frequency component appears in the measured result, as shown in figure 21, it is impossible to distinguish whether it is caused by the master gear or by the gear-measuring instrument. The wedge artefact has a measurement plane with low roughness and few undulation. Thus, it can be judged that the high-frequency component observed in the measurement result in figure 21 is caused by the gear-measuring instrument.

In figure 20, the absolute value of the actually measured curve appears to be slightly smaller than that of the theoretical curve, although the shapes of the two curves are almost the same. As a result, the deviation curve, which is obtained by subtracting the theoretical curve from the measured curve, has a reverse shape compared with the theoretical curve. Such an undulation does not exist on the lead measurement plane of the wedge artefact and thus it can be judged that this deviation is caused by the errors of gear-measuring 
instrument. For instance, the sensitivity error of the displacement sensor is one of the error factors that cause such a phenomenon. That is, it is possible that the sensitivity coefficient is slightly small in this measuring instrument. The amplitude of the deviation between the theoretical and measured curves is about $5 \mu \mathrm{m}$ for a displacement of $100 \mu \mathrm{m}$; in other words, the sensitivity error is estimated to be $5 \%$. The master gear or the lead master has a reference surface with a similar shape to the theoretical helicoids, and therefore the displacement detected by the sensor is very small when the master gear or the lead master is measured. In this case, a deviation of only about several hundreds of nanometers will appear in the measured result due to the sensitivity error of $5 \%$. Therefore, even if there is a sensitivity error, it is difficult to detect it using the master gear or the lead master. On the other hand, it is easy to detect the sensitivity error when the wedge artefact is measured because it uses the wide measurement range of the displacement sensor. This is one of the advantages of the wedge artefact.

\subsection{Features improved by wedge artefact and remaining problem}

In this section, the features improved by wedge artefact and a remaining problem are discussed by comparing with those obtained by the conventional master gear or lead master.

The conventional master gear is manufactured by grinding, and thus, deep grinding marks exist on the tooth flank. Therefore, roughness and undulation appear on the tooth flank to a certain degree. We previously determined that the minimum deviation between the theoretical involute helicoid and the actual tooth flank of the master gear is $1 \mu \mathrm{m}$ owing to roughness and undulation, even if a high-precision process is performed. When the master gear is measured, the roughness and undulation cause high- and low-frequency waved forms in a measured curve. Therefore, when a gear-measuring instrument is evaluated using the master gear, it is difficult to determine whether the waved forms in the measured curve, whose amplitude is below $1 \mu \mathrm{m}$, are caused by the gear-measuring instrument or master gear.

On the other hand, the lead measurement plane of wedge artefact can be polished after grinding. Thus, the roughness and undulation of the surface are small. A plane with an undulation of $0.03 \mu \mathrm{m}$ can be realized if a suitable material and process are chosen, although the artefact manufactured in this study has an undulation of $0.5 \mu \mathrm{m}$. Therefore, when the wedge artefact is measured to evaluate the gear-measuring instrument, it is possible to determine whether the waved forms in the measured curve, whose amplitude is from sub-micrometer to $1 \mu \mathrm{m}$, are caused by the measuring instrument; however, it is impossible when the master gear is used. This is a feature improved by the wedge artefact compared with the conventional master gear.

On the contrary, the setting error (alignment error) of the wedge artefact on the gear-measuring instrument is a problem of wedge artefact. The conventional master gear also has the same problem. The setting error of wedge artefact makes a measured curve inclined. For example, suppose that the wedge artefact inclines around the $\mathrm{x}$-axis by $0.44 \times 10^{-3}$ degrees (inclination of $1 \mu \mathrm{m}$ per $130 \mathrm{~mm}$ (length of plane $\mathrm{S}_{2}$ )). Under such a condition, the measured curve inclines by $0.54 \mu \mathrm{m}$ per measurement range in the tooth width direction of 50 $\mathrm{mm}$ compared with the original curve without the inclination of the wedge artefact. Thus, it is difficult to determine whether the inclination of the measured curve is caused by the gear-measuring instrument or the 
setting error of wedge artefact. This problem remains even if the wedge artefact is used.

\section{Conclusion}

The tooth lead measurement function of a gear-measuring instrument must be evaluated with high accuracy. However, the current master gear or lead master used for the evaluation of the gear-measuring instrument does not satisfy the required accuracy. To solve this problem, a wedge artefact is proposed to evaluate the lead measurement, which is composed of planes and can be produced with high accuracy. The following results were achieved in this study.

(1)A mathematical model of the situation where the wedge artefact is measured on the gear-measuring instrument was constructed. A virtual measurement result can be obtained using the model, assuming that the wedge artefact is measured by the gear-measuring instrument without any errors.

(2)A method to design the wedge artefact was proposed.

(3)The wedge artefact was produced and was calibrated. It was confirmed that the lead measurement plane has high flatness with low roughness and few undulations.

(4)A measurement procedure for the wedge artefact was proposed, and it was confirmed that the evaluation of the gear-measuring instrument using the wedge artefact was effective by performing a measurement experiment.

Because this wedge artefact is composed of planes, it can be produced with high accuracy and can also be calibrated using a measuring machine with small uncertainty. This leads to the small uncertainty of the artefact. Because roughness and undulation of the lead measurement plane of wedge artefact can be several tens of nanometers, error of gear-measuring instrument which causes waved form in measured curve can be found with accuracy of hundreds of nanometers. In addition, the wedge artefact has an advantage that it can detect the sensitivity error of the displacement sensor of the gear-measuring instrument because it uses the wide measurement stroke of the displacement sensor. On the basis of these results, it is thought that the wedge artefact can contribute to improving the accuracy of the evaluation of the lead measurement.

\section{Acknowledgements}

This research is supported by a Grant-in-Aid for Scientific Research (B) and by the members of the Japanese Gear Manufacturers’ Society (JGMA) committee on the tooth lead standard.

\section{References}

[1] Komori M, Kubo A and Kawasaki Y 2000 Design Method of Vibrationally Optimum Tooth Flank Form for Involute Helical Gears with Scattering in Pressure Angle and Helix Angle Deviation, Proc. of DETC2000 ASME 8th International Power Transmission and Gearing Conf. (Baltimore) DETC2000/PTG-14423

[2] Komori M, Kubo A and Suzuki Y 2003 Simultaneous Optimization of Tooth Flank Form of Involute Helical Gears in Terms of Both Vibration and Load Carrying Capacity Japan Society of Mechanical Engineers International Journal C46(4) pp.1572-1581 
[3] Goch G 2003 Gear Metrology CIRP Annals - Manufacturing Technology 52(2) pp.659-695

[4] Robinson M J, Oakley J P and Cunningham M J 1995 The Accuracy of Image Analysis Methods in Spur Gear Metrology Measurement Science and Technology 6(7) pp.860-871

[5] Suh S H, Lee E S, Kim H C, and Cho J H 2002 Geometric Error Measurement of Spiral Bevel Gears Using a Virtual Gear Model for STEP-NC International Journal of Machine Tools and Manufacture 42(3) pp.335-342

[6] Boukebbab S, Bouchenitfa H, and Boughouas H, Linares J M 2007 Applied iterative closest point algorithm to automated inspection of gear box tooth Computers \& Industrial Engineering 52(1) pp.162-173

[7] Guenther A and Hocken R 2006 Evaluation of Runout Deviation at Bevel Gears based on Pitch Measurements CIRP Annals - Manufacturing Technology 55(1) pp.539-542

[8] Dantan J Y, Bruyere J, Baudouin C, and Mathieu L 2007 Geometrical Specification Model for Gear Expression, Metrology and Analysis CIRP Annals 56(1) pp.517-520

[9] Oguma T and Kawasaki Y 2001 History and Prospect of Gear Measuring Instrument Proc. of MPT 2001 JSME International Conf. on Motion and Transmissions (Fukuoka) pp.623-629

[10] Rasnick B, Cox B and Sherrill M 1998 Determination of Gear Artifact Measurement Uncertainty on Coordinate Measurement Machines by Measurement Decomposition and Utilization of Reference Artifacts Proc. of Measurement Science Conf. (Pasadena)

[11] Fujio H, Kubo A, Tochimoto S, Hanaki H, Saito Y and Suzuki M 1992 Measurement of Three-Dimensional Curved Surface of Machine Part Using Holographic Interferometry : 1st Report, Correspondence between Coordinate System of Measured Surface and That of its Image Transactions of the Japan Society of Mechanical Engineers. C 58(545) pp.295-301

[12] Okuyama E, Kiyono S and Moritoki H 1994 Investigation of an Optical Noncontact Gear Geometry Measurement System: Measurement of Pitch Errors and Tooth Profiles Precision Eng. pp.117-123

[13] Haertig F, Keck C, Kniel K, Schwenke H I, Waeldele F and Wendt K 2005 A Novel Concept for High Accuracy Gear Calibration Gear Technology 22(3) pp16-20

[14] Haertig F, Keck C, Kniel K, Schwenke H I, Wendt K and Waeldele F 2003 Development of a Novel Gear Measuring Device of High Accuracy Proc. of DECT'03 ASME 2003. (Chicago) 22(3) PTG-48105

[15] Bicker R, Frazer R C and Wehmeyer D 2001 Verifying Position Errors in CNC Gear Measuring Instruments using a Laser Interferometer with Dynamic Data Capture Software Laser Metrology and Machine Performance V pp.345-354

[16] Physikalisch-Technische Bundesanstalt brochure Gear Metrology in the PTB

[17] Och R 2006 History of Gear Measurement - Measuring Machines and Traceability 1900-2005 - Gear Product News Oct.

[18] Beyer W 1996 Traceable Calibration of Master Gears at PTB Technical Paper of American Gear Manufacturers Association (Alexandria) 96FTM04

[19] Beyer W and Pahl W 1996 Advanced Strategies for the Traceable Calibration of Cylindrical Gears and Bebel Gears at PTB VDI- BERICHTE NR 1230 pp.937-945 
[20] Frazer R C, Bicker R, Cox B, Harary H and Haertig F 2004 An International Comparison of Involute Gear Profile and Helix Measurement Metrologia pp.12-16

[21] Kubo A, Kondo K, Takatsuji T, Komori M, Osawa S and Naoi K 2006 Coming Japanese Industrial Standard Calibration System of Involute Form Checker Proc. of International Conf. of Metrology (Casablanca)

[22] Innocenti C 2007 Simple Techniques for Measuring the Base Helix Angle of Involute Gears Proc. of 12th IFToMM World Congress (Besancon) No.406

[23] Sammartinia M P and De Chiffre L 2000 Development and Validation of a New Reference Cylindrical Gear for Pitch Measurement Precision Eng. 24(4) pp.302-309

[24] Takeoka F, Komori M, Kubo A, Fujio H, Taniyama S, Ito T, Takatsuji T, Osawa S and Sato O 2008 Design of Laser Interferometric Measuring Device of Involute Profile J. of Mechanical Design 130(5) pp.052602

[25] Kondo K and Mizutani H 2002 Measurement Uncertainty of Tooth Profile by Master Balls VDI-BERICHTE NR.1665 pp.797-810 


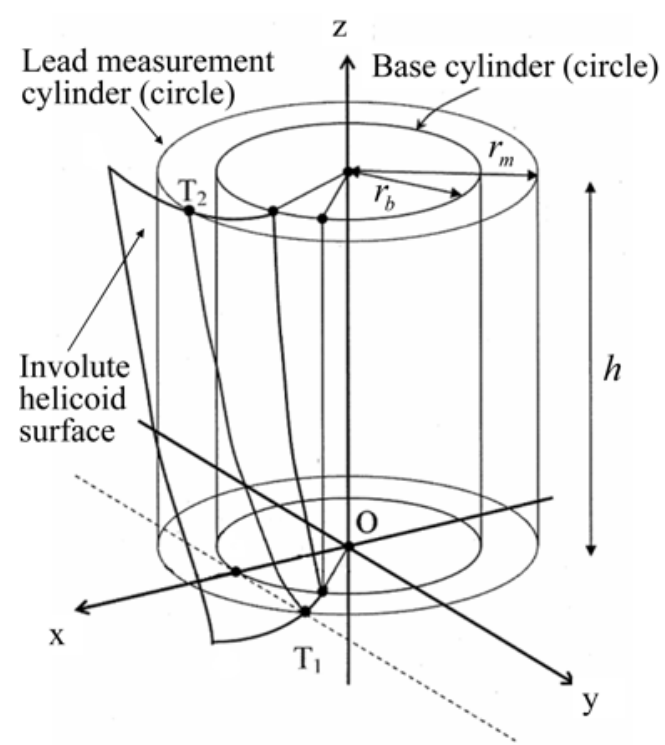

Figure 1. Base cylinder (circle), involute helicoid surface, and lead measurement cylinder (circle) of measured gear.

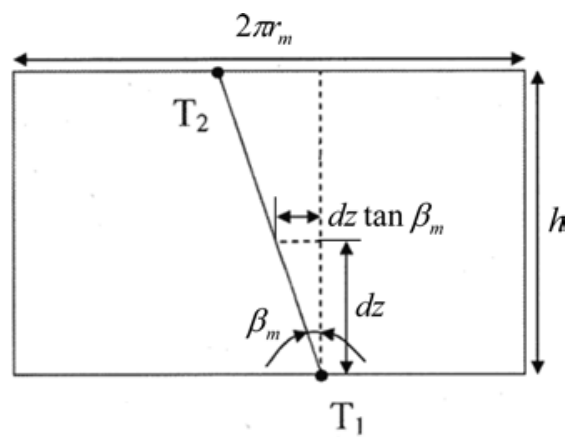

Figure 2. Development surface of lead measurement cylinder.

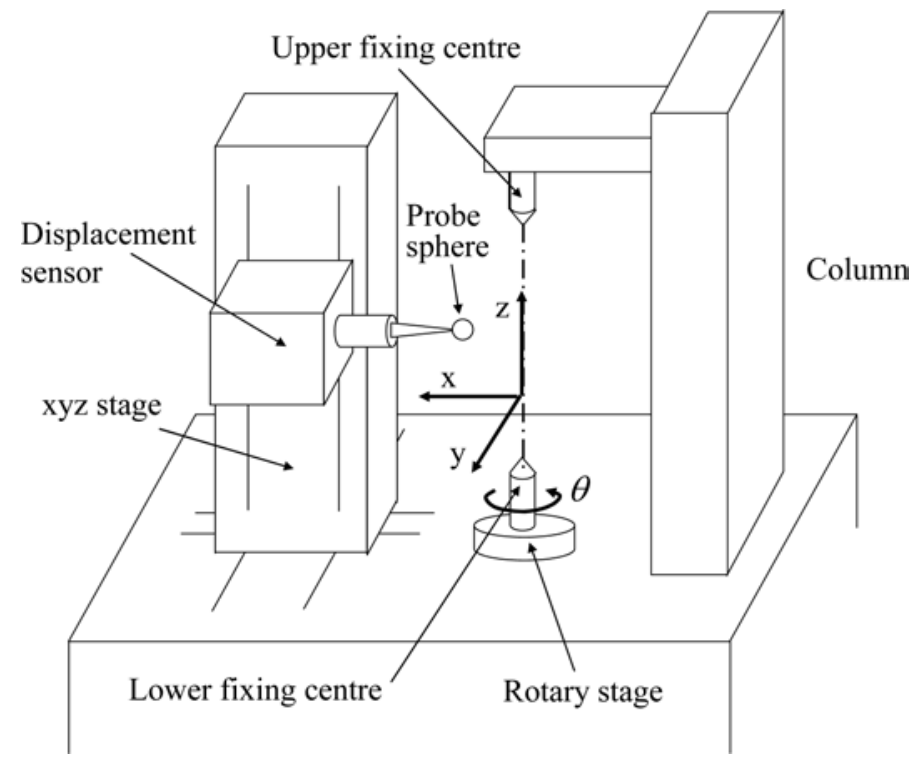

Figure 3. Schematic model of gear-measuring instrument. 


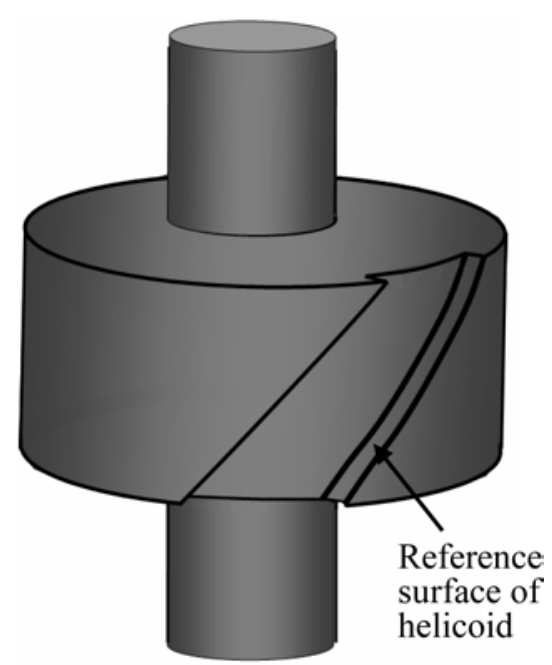

Figure 4. Conventional lead master.
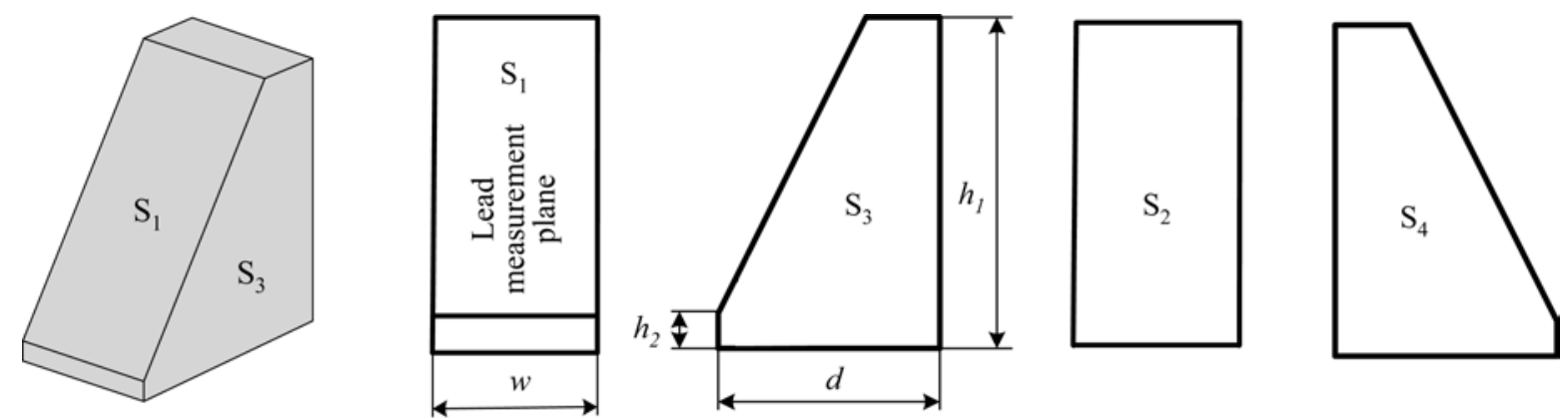

Figure 5. Schematic diagram of proposed wedge artefact. 


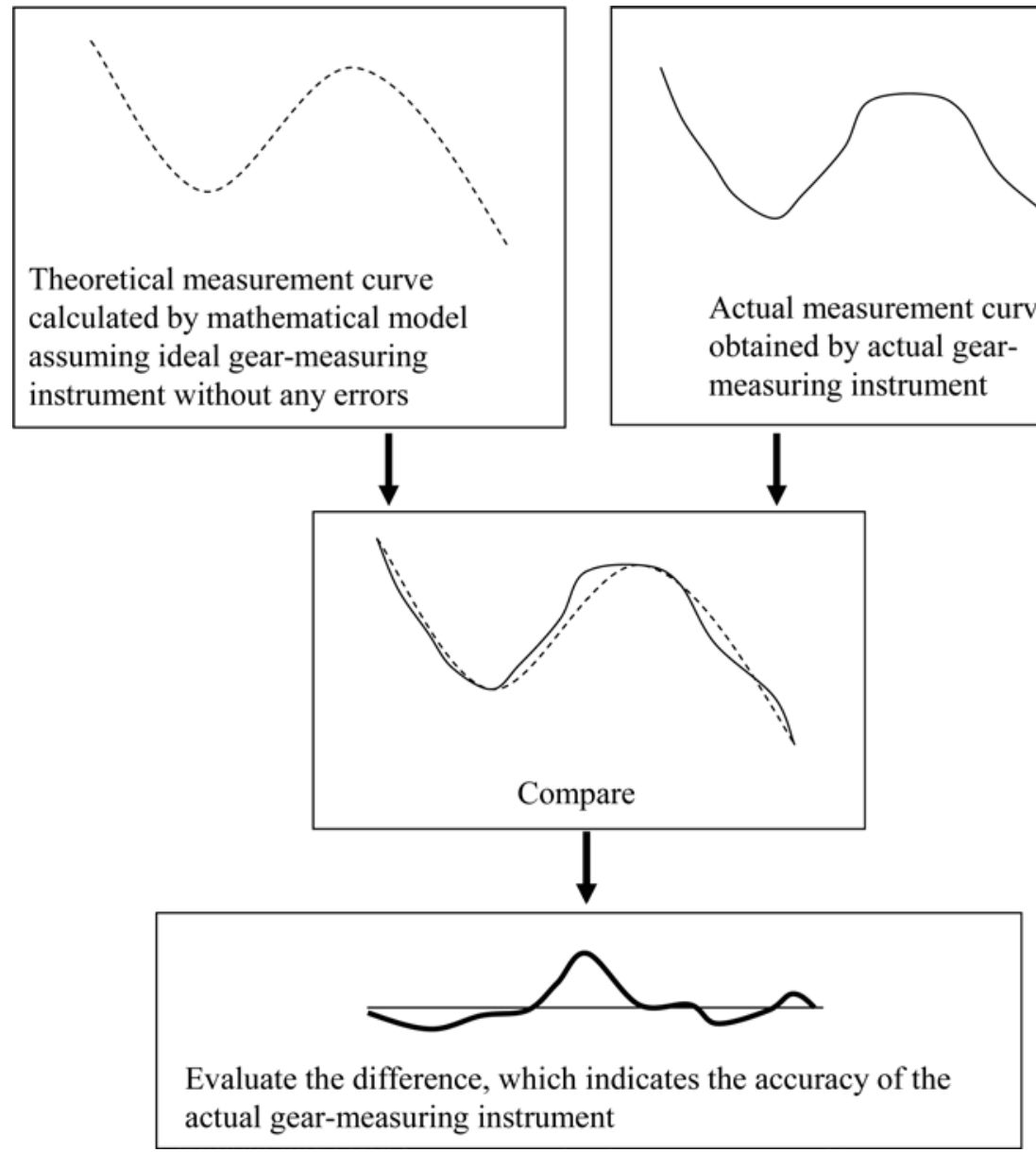

Figure 6. Flowchart used to evaluate the accuracy of gear-measuring instrument using the wedge artefact.

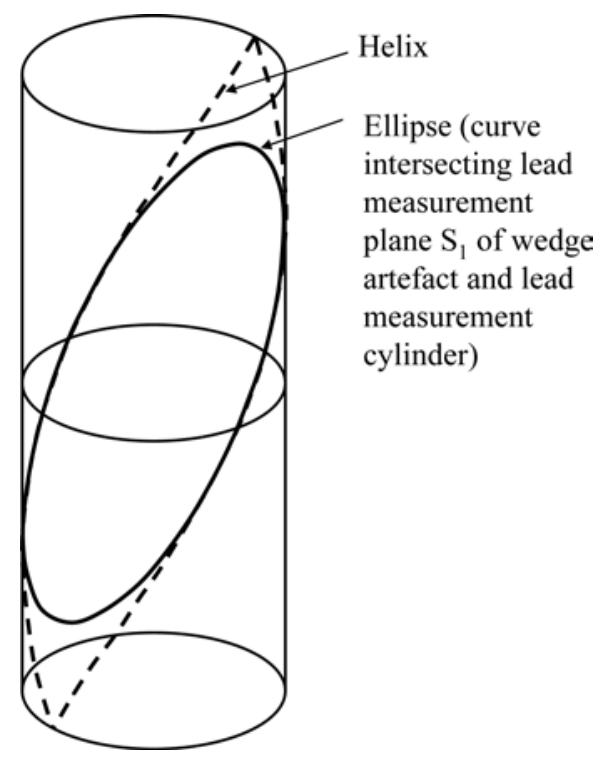

Figure 7. Helix and ellipse (curve intersecting lead measurement plane and cylinder) on the lead measurement cylinder. 


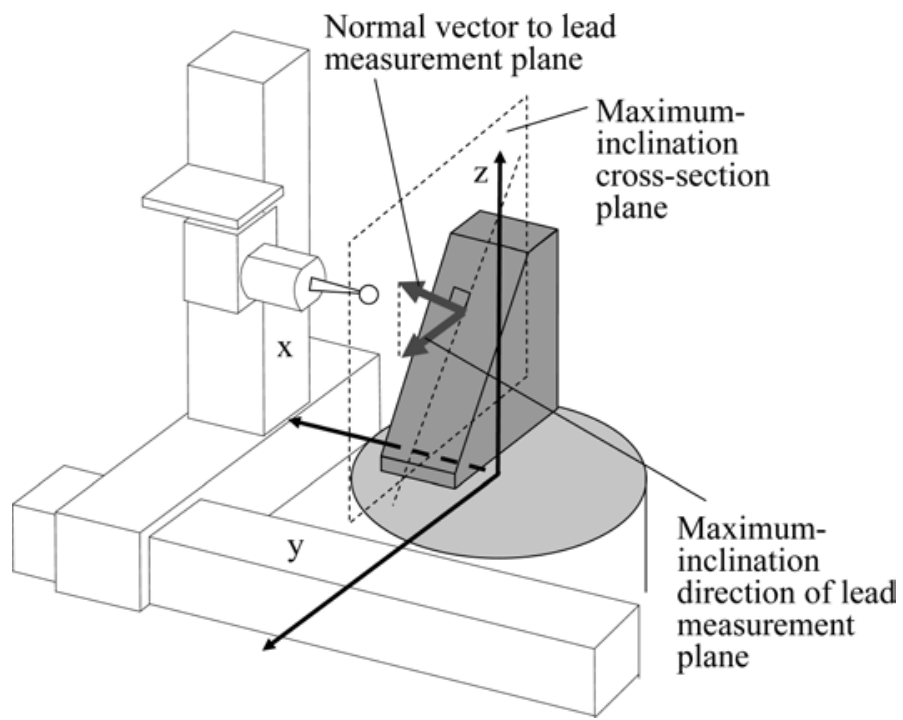

Figure 8. Basic posture of wedge artefact on gear-measuring instrument.

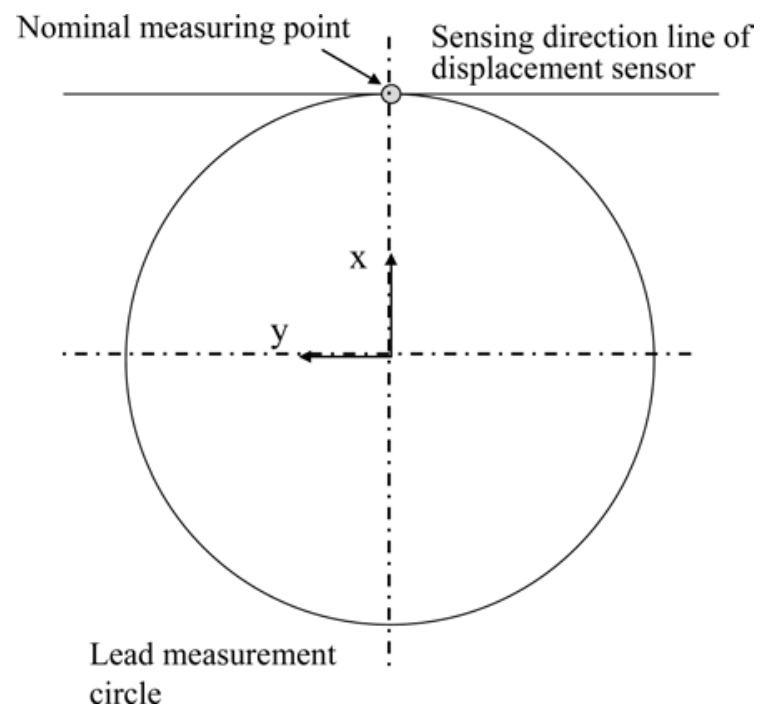

Figure 9. Lead measurement position. 


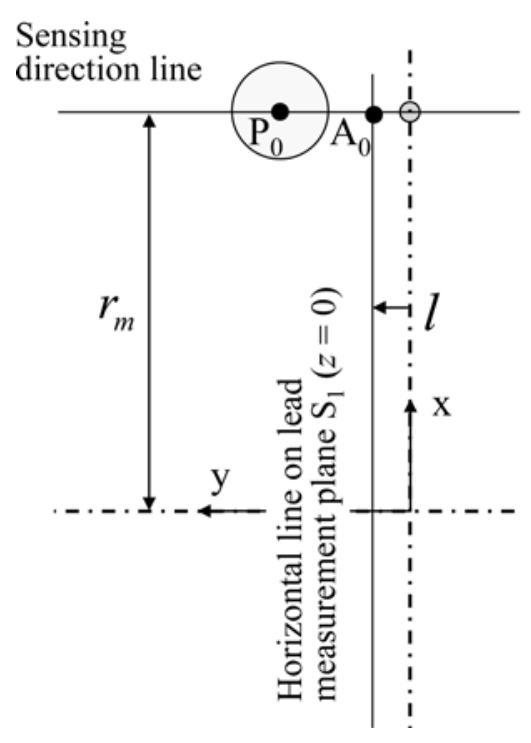

(a) Cross section at $z=0$

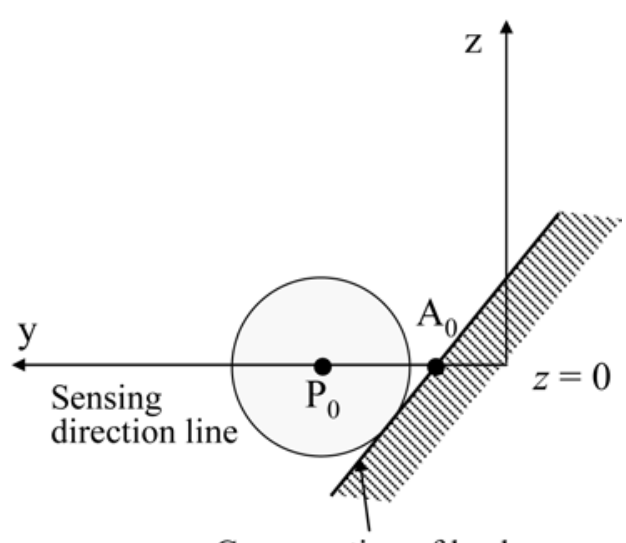

Cross section of lead measurement plane $\mathrm{S}_{1}$

Figure 10. Probe sphere in contact with lead measurement plane $S_{1}$ of wedge artefact in the basic posture on gear-measuring instrument.
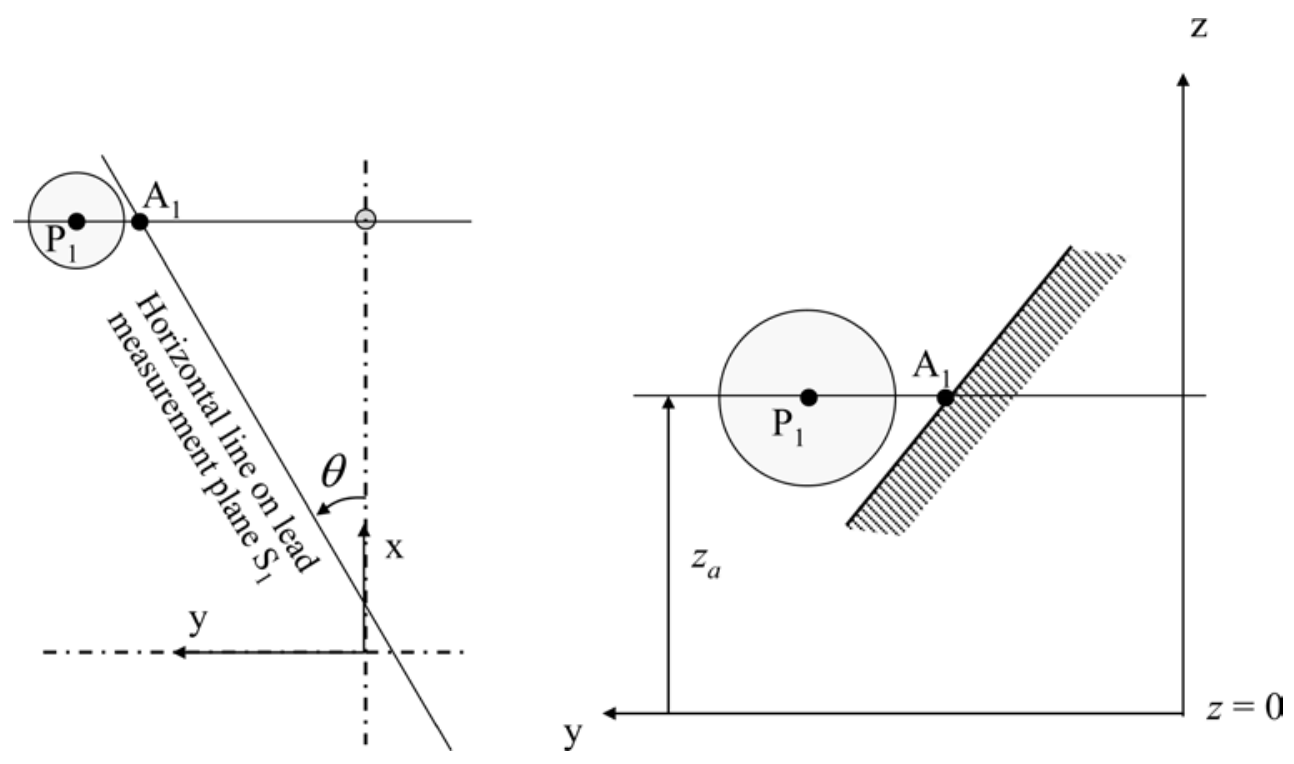

(a) Cross section at $z=Z_{a}$

(b) Cross section at $x=r_{m}$

Figure 11. State after rotary stage has rotated by $\theta$. 


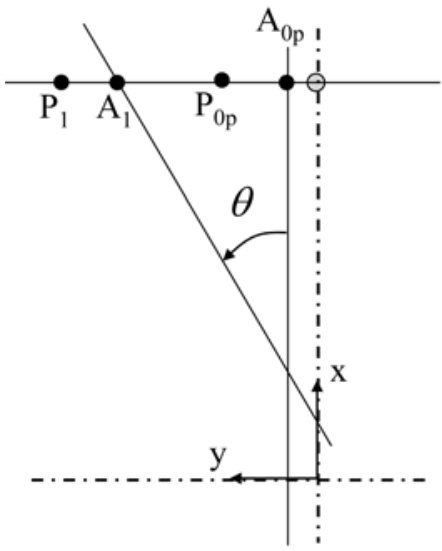

(a) Cross section at $z=Z_{a}$

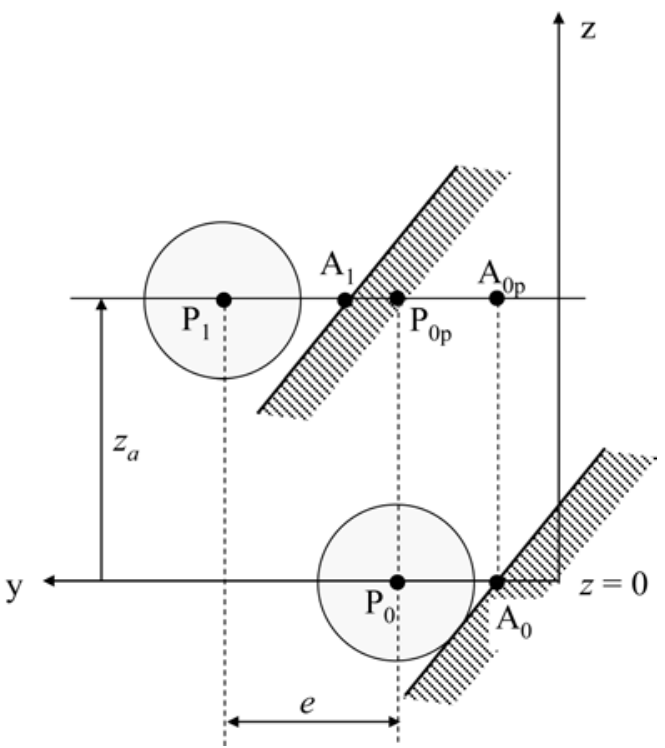

(b) Cross section at $x=r_{m}$

Figure 12. State in basic posture and that after rotation of wedge artefact.
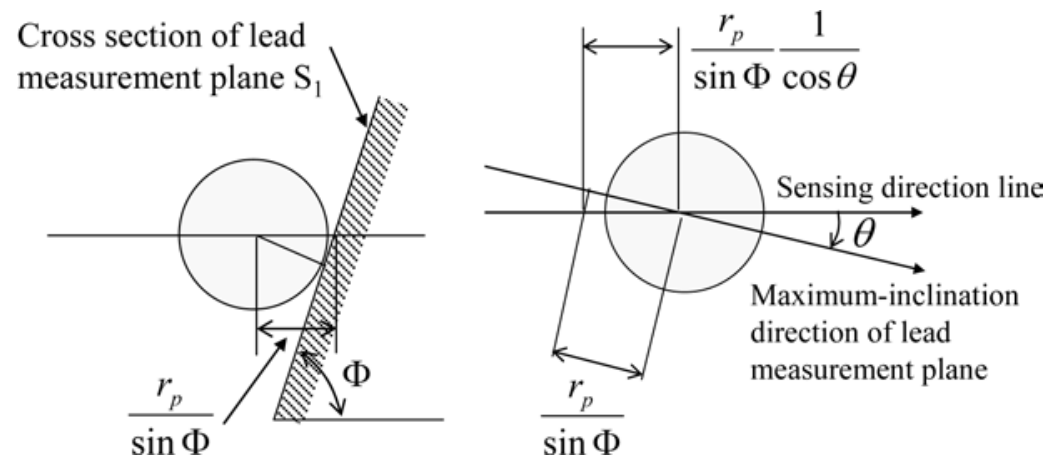

(a) Maximum-inclination cross-section plane passing through the centre of probe sphere

(b) Horizontal cross section including centre of probe sphere

Figure 13. Contact condition of probe sphere. 

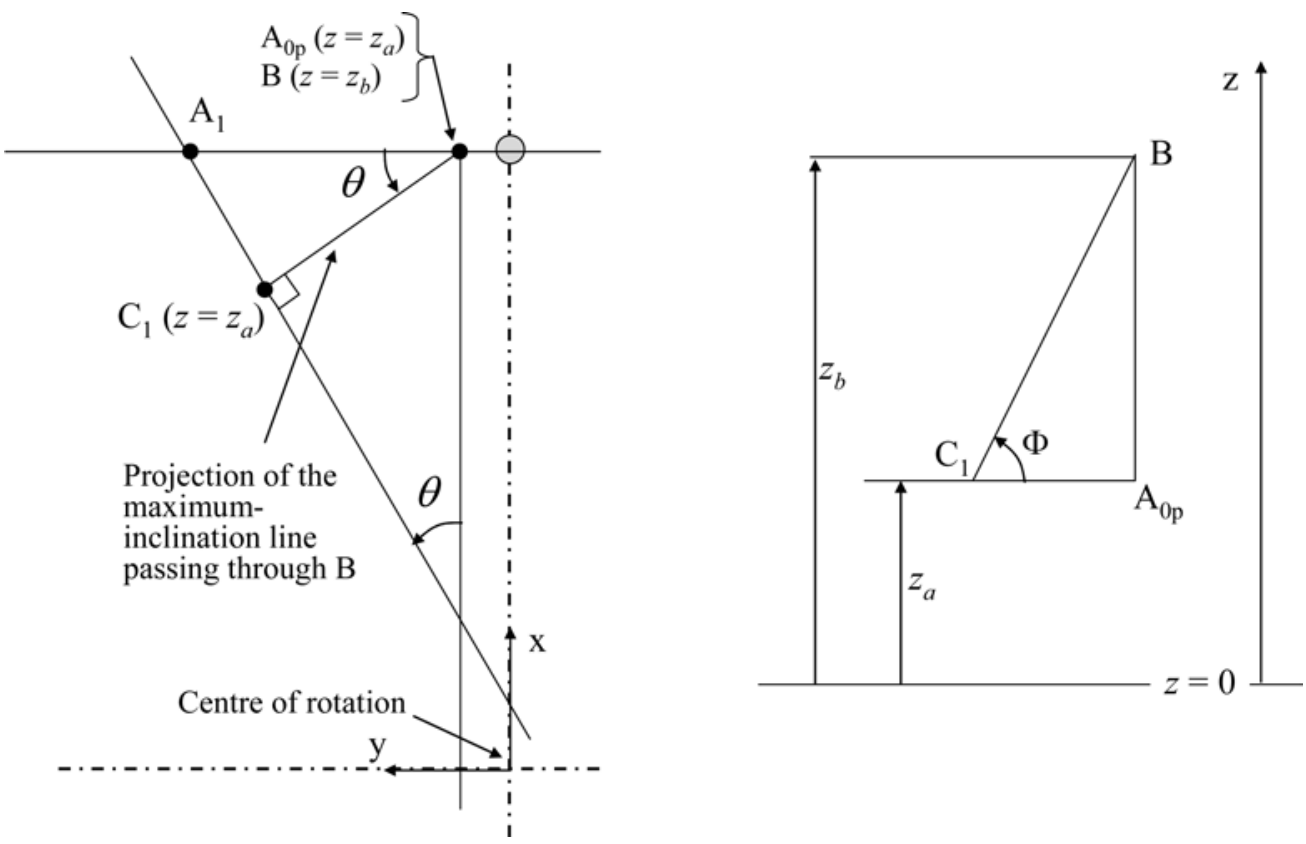

(a) Horizontal cross section at $z=z_{a}$ (point $B$ is projected onto the plane $z=z_{a}$ )

(b) Maximum-inclination cross-section plane passing through $\mathrm{B}$ and $\mathrm{C}_{1}$

Figure 14. Comparison between initial condition and that after rotation of $\theta$.

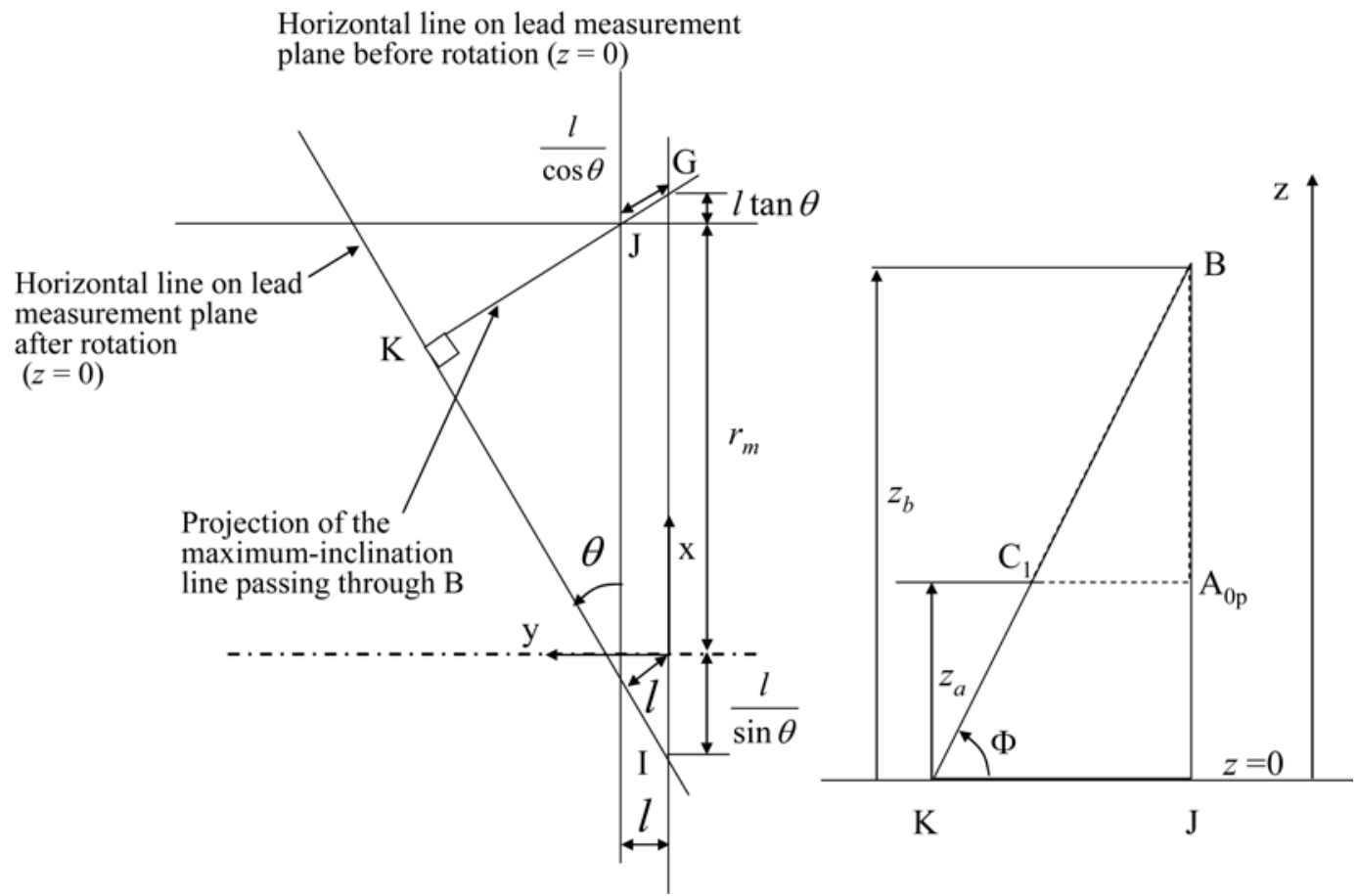

$\begin{array}{ll}\text { (a) Horizontal view at } \mathrm{z}=0 & \text { (b) Vertical cross section (maximum-inclination cross-section plane) }\end{array}$

Figure 15. Condition at $\mathrm{z}=0$ for calculation of $z_{b}$. 


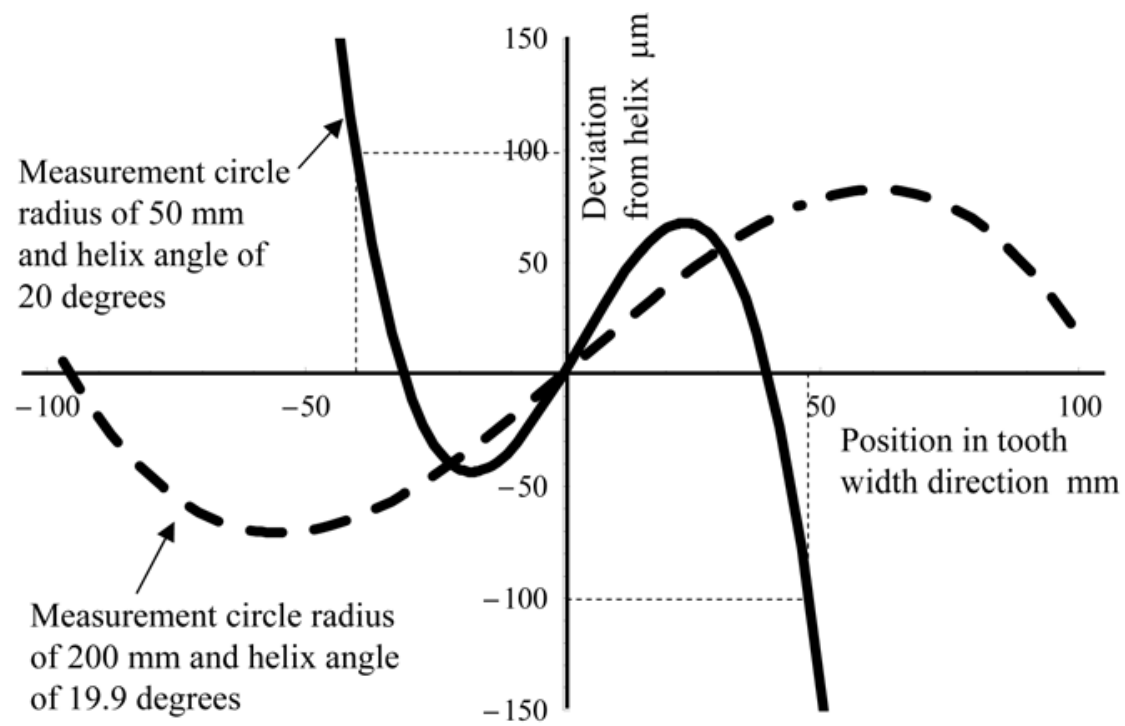

Figure 16. Virtual measurement curve (theoretical measurement curve) for tooth lead measurement of wedge artefact, which is calculated using the mathematical model (the inclination angle of the lead measurement plane of wedge artefact is 70.2 degrees and the radius of probe sphere is $1 \mathrm{~mm}$ ).

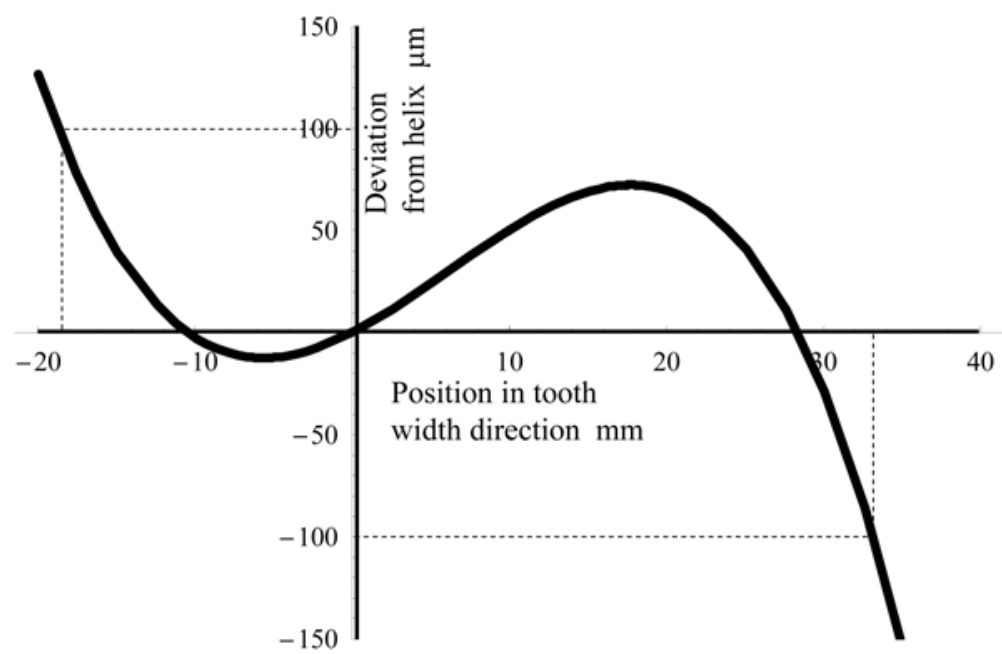

Figure 17. Virtual measurement result (theoretical measurement curve) of lead using designed wedge artefact, where the radius of the measurement circle is $49 \mathrm{~mm}$, the helix angle on the measurement cylinder is 30 degrees, and the radius of the probe sphere is $3 \mathrm{~mm}$. 


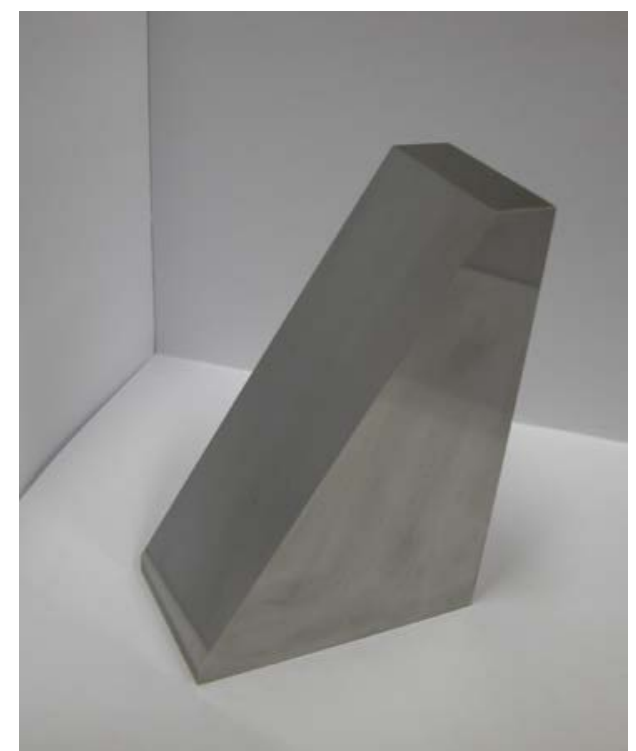

Figure 18. Produced wedge artefact.

Table 1. Flatness of each plane of wedge artefact.

\begin{tabular}{|c|c|c|c|}
\hline $\mathrm{S}_{1}$ & $\mathrm{~S}_{2}$ & $\mathrm{~S}_{3}$ & $\mathrm{~S}_{4}$ \\
\hline $0.87 \mu \mathrm{m}$ & $6.77 \mu \mathrm{m}$ & $3.78 \mu \mathrm{m}$ & $5.99 \mu \mathrm{m}$ \\
\hline
\end{tabular}
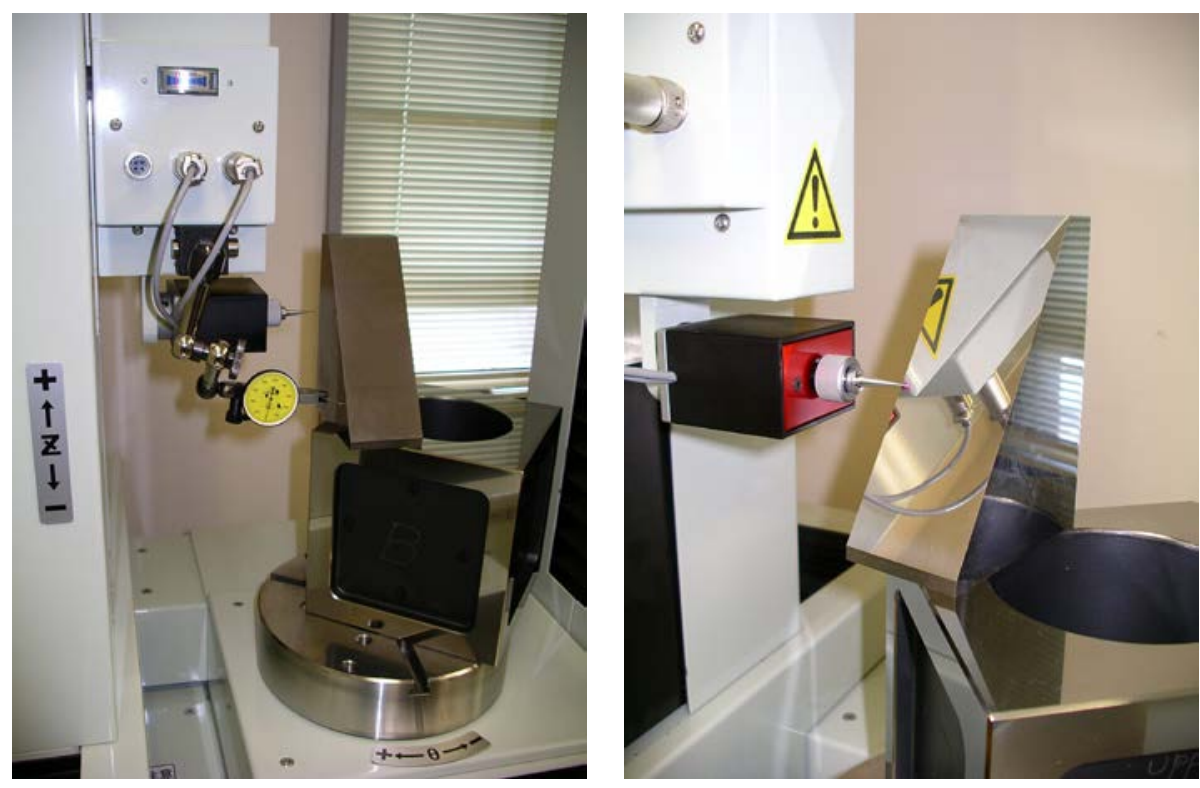

(a) Measurement of plane $\mathrm{S}_{4}$ using dial gauge to realize basic posture

(b) Measurement of plane $\mathrm{S}_{1}$

Figure 19. Setting and measurement of wedge artefact using gear-measuring instrument. 
Table 2. Parameters in measurement experiment.

\begin{tabular}{|l|c|c|c|c|c|c|c|c|c|}
\hline No. & 1 & 2 & 3 & 4 & 5 & 6 & 7 & 8 \\
\hline $\begin{array}{l}\text { Radius of lead measurement } \\
\text { circle } r_{m}(\mathrm{~mm})\end{array}$ & \multicolumn{7}{|c|}{4} & \multicolumn{2}{|c|}{4} \\
\hline $\begin{array}{l}\text { Helix angle on lead } \\
\text { measurement cylinder } \beta_{m} \\
\text { (degree) }\end{array}$ & 30.05 & 30.00 & 29.95 & 29.90 & 29.85 & 29.80 & 29.75 & 30.00 \\
\hline
\end{tabular}

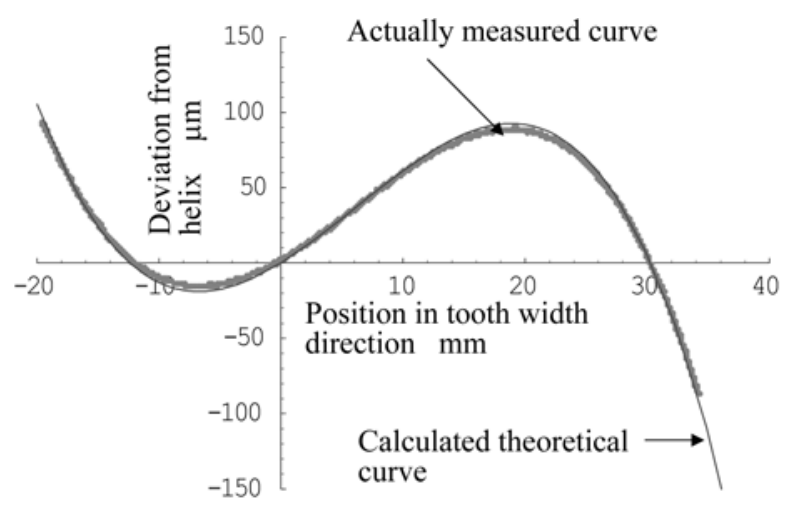

Figure 20. Calculated theoretical curves and actually measured curves of experiment No.1.

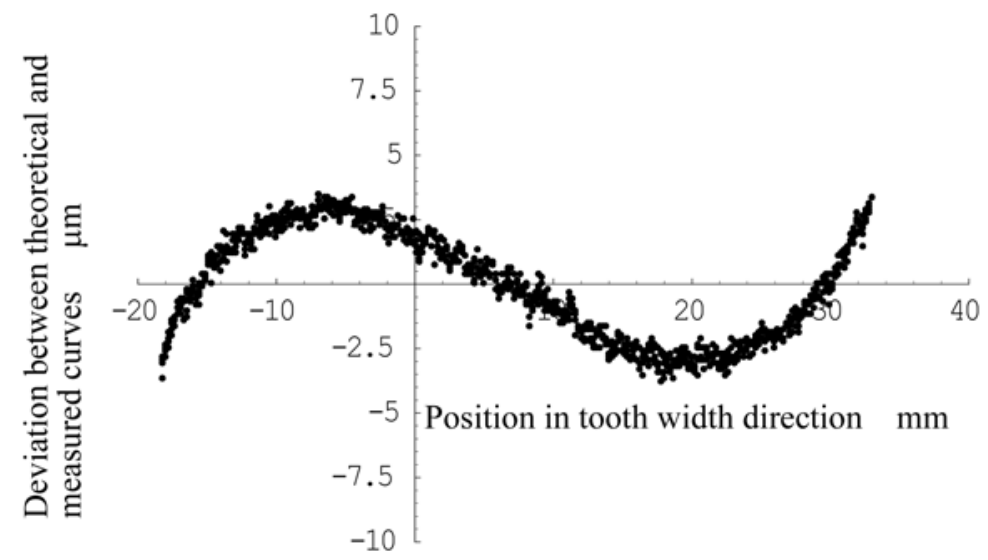

Figure 21. Deviations between theoretical and measured curves of experiment No.1. 